\title{
A Genome-Wide Association Study Identifies a Novel Susceptibility Locus at The DLGAP1 Gene For Resistant Hypertension in The Japanese Population
}

\author{
Yasuo Takahashi ( $\nabla$ takahashi.yasuo@nihon-u.ac.jp ) \\ Nihon University School of Medicine \\ Keiko Yamazaki \\ RIKEN Center for Integrative Medical Sciences \\ Yoichiro Kamatani \\ University of Tokyo \\ Michiaki Kubo \\ RIKEN Center for Integrative Medical Sciences

\section{Koichi Matsuda} \\ University of Tokyo
}

\section{Satoshi Asai}

Nihon University School of Medicine

\section{Research Article}

Keywords: resistant hypertension, genome-wide association study, DLGAP1, aldosterone synthase gene, CYP11B2

Posted Date: January 18th, 2021

DOI: https://doi.org/10.21203/rs.3.rs-143274/v1

License: (c) (i) This work is licensed under a Creative Commons Attribution 4.0 International License. Read Full License 


\section{Abstract}

Numerous genetic variants associated with hypertension and blood pressure are known, but there is a paucity of evidence from genetic studies of resistant hypertension, especially in the Asian populations. To identify novel genetic loci associated with resistant hypertension in the Japanese population, we conducted a genome-wide association study with 2,705 resistant hypertension cases and 21,296 mild hypertension controls, all from BioBank Japan. We identified one novel susceptibility locus, rs1442386 on chromosome 18p11.3 (DLGAP1), achieving genome-wide significance (odds ratio $(95 \% \mathrm{Cl})=0.85(0.81-0.90), P$ $=3.75 \times 10^{-8}$ ) and 17 loci showing suggestive association, including rs62525059 of 8q24.3 (CYP11B2). We further detected biological processes associated with resistant hypertension, including chemical synaptic transmission, regulation of transmembrane transport, neuron development and neurological system processes, highlighting the importance of the nervous system. This study provides insights into the etiology of resistant hypertension in the Japanese population.

\section{Introduction}

Resistant hypertension is a medical disorder in which patients require at least four antihypertensive drugs of different classes for blood pressure control1,2, and is an increasingly important clinical problem. The exact prevalence of resistant hypertension is unknown. Data from clinical trials, however, suggest that it is not uncommon, affecting about 20 to $30 \%$ of patients with hypertension3, 4. In the US National Health and Nutrition Examination Survey (NHANES), the prevalence of uncontrolled hypertension despite use of at least three antihypertensive drugs was reported to increase from $16 \%$ of patients treated for hypertension in 1998-2004 to $28 \%$ in 2005-20085. Also, Sarafidis et al recently estimated that the prevalence of resistant hypertension is $8-12 \%$ of adult patients with hypertension, using data from NHANES6.

The etiology of resistant hypertension is unknown, but appears to be multifactorial7, with lifestyle-related, hormonal, and genetic factors having possible important roles. In addition to previously known risk factors, including older age and obesity, medical conditions such as impaired renal function and diabetes mellitus are also associated with resistant hypertension4,8-10. Elevation of circulating aldosterone level has been identified in the majority of patients with resistant hypertension 11-13, drawing attention to the importance of aldosterone in the pathogenesis of resistant hypertension14. Also, genetic factors are believed to play a role in the disease etiology 15. The speculation that the role of genetic factors may be greater in patients with resistant hypertension than in those with general hypertension would not be inconsistent with excessive blood pressure phenotypes of resistant hypertension. Although previous studies have identified numerous genetic variants associated with hypertension and blood pressure16-19, there is a paucity of evidence from genetic studies of resistant hypertension. The available genetic data regarding resistant hypertension are limited and primarily focused on candidate genes20-25. Additionally, pharmacogenomics research on resistant hypertension is in progress 15. Recently, a few studies with a comprehensive genetic approach, genome-wide association studies (GWASs), have identified some significant loci for susceptibility to resistant hypertension in the US population26-28. However, there is still little evidence from genome-wide investigations of resistant hypertension, especially in the Asian population. Therefore, we performed a GWAS to identify novel genetic loci associated with resistant hypertension in the Japanese population.

\section{Materials And Methods}

\section{Data source}

The genotyping data and clinical information in this GWAS were obtained from BioBank Japan. The BioBank Japan project, which began in 2003, is a collaborative network of 66 hospitals in all areas of Japan that has collected genomic DNA, serum and clinical information from approximately 270,000 patients diagnosed with any of 51 diseases 45,46 . All subjects received a detailed explanation, and all signed a written informed consent form. The study protocol conformed to the ethical guidelines of the Declaration of Helsinki and was approved by the Ethics Committees of all participating institutions, including the Institute of Medical Science, the University of Tokyo, the Center for Integrative Medical Sciences, RIKEN, and Nihon University School of Medicine. 


\section{Study populationsand phenotype definitions}

First, we identified 78,463 Japanese patients with hypertension who participated in the BioBank Japan project, using drug prescription data, fulfilling the following criteria: 1) Patients who had received at least one prescription of any antihypertensive drug between 2003 and 2012, as listed in Supplementary Table S10. Direct alpha antagonists such as phentolamine and phenoxybenzamine were excluded from this drug list because they are mainly used for the treatment of pheochromocytoma and hypertensive emergencies 26 .

Second, we selected study subjects, aged over 40 years, with resistant hypertension as cases and mild hypertension as controls, fulfilling the following criteria: 1) The resistant hypertension group was defined as patients who had received four or more classes of antihypertensive drugs for at least one year, whatever their blood pressure. 2) The control group was defined as patients with mild hypertension who had received one antihypertensive drug for at least one year. Patients who had received at least one prescription of two or more antihypertensive drugs were excluded from the control group. Consequently, we identified a total of 25,450 patients with hypertension (3,978 cases and 21,472 controls) who fulfilled the above criteria, and their prescription rates for each class of antihypertensive drugs are listed in Supplementary Table S9. After quality-control and PCA described in detail below, 2,705 cases and 21,296 controls were used to perform a GWAS. The characteristics of the study subjects are summarized in Table 2.

\section{Genotyping and imputation}

Genotyping was performed using the Illumina HumanOmniExpressExome BeadChip, or Illumina HumanOmniExpress and HumanExome BeadChip. We aligned the probe sequence in the manifest files of the genotyping array to the GRCh37.3 reference using BLAST to convert genotypes into forward strands. For sample quality control, we excluded samples with (i) sample call rate $<0.98$, (ii) closely related samples identified using identity by state (IBS) using PLINK47, and (iii) outliers from the East Asian Cluster using PCA for genotype by smartpca48. Then, we applied quality control for genetic variants and excluded SNPs with (i) SNP call rate $<0.99$ in both cases and controls, (ii) Hardy-Weinberg equilibrium $P \leq 1 \times 10^{-6}$ and MAF $<1 \%$. A Q-Q plot was constructed using observed $P$ values against expected $P$ values and an inflation factor value ( $\lambda$-value) that was calculated to assess potential population stratification of the study subjects 49 .

We pre-phased the genotypes with SHAPEIT50 and imputed dosages with IMPUTE251 using the 1000 Genomes Projects Phase III as a reference52, which was supplied by IMPUTE2 site. For subsequent analysis, we used genotypes with an imputation quality of info $\geq 0.8$ and $M A F \geq 5 \%$.

\section{Analysis of GWAS data}

For general statistical analysis, we used R statistical environment version 3.4.3 or PLINK1.07. GWAS was used to perform association analysis using imputed allele dosages by snptest53. We set the threshold for genome-wide significance at the level of $P<5 \times 10^{-8}$ and the threshold for suggestive significance at $P \leq 1 \times 10^{-5}$. A Manhattan plot was generated using $\mathrm{R}$ software to visualize the results. Regional association plots were generated using LocusZoom54. The online tool HaploReg v4.1 (https://pubs.broadinstitute.org/mammals/haploreg/haploreg.php) was used to explore the genes nearest to the index SNPs, and genes containing a missense mutation in high linkage disequilibrium (LD) ( $r 2>0.8$ ) with the GWAS SNPs55. The effects of GWAS SNPs on expression in eQTL studies of different tissues were extracted from the query results of HaploReg.

We defined an associated locus as a genomic region within $\pm 1 \mathrm{Mb}$ from the lead SNP. We defined a locus as novel when it did not include any variants that were previously reported to be significantly associated with blood pressure phenotypes, hypertension, or resistant hypertension ( $P$ in previous GWAS $\left.<5.0 \times 10^{-8}\right)$.

\section{Replication of previously reported variants by this GWAS}

To verify previously reported variants showing an association $\left(P<1 \times 10^{-5}\right)$ with resistant hypertension in a multi-ethnic GWAS dataset26-28,31, we evaluated 26 SNPs in the present Japanese GWAS dataset. Also, we further examined SNPs, previously 
associated with hypertension or blood pressure phenotypes, in the current GWAS dataset. From the NHGRI European Bioinformatics Institute (NHGRI-EBI) GWAS Catalogue (https://www.ebi.ac.uk/gwas/, accessed July 2020), 172 and 2074 variants showing an association $\left(P<5 \times 10^{-8}\right)$ with hypertension and blood pressure phenotypes (including blood pressure, systolic blood pressure, diastolic blood pressure, and mean arterial pressure), respectively were obtained. We then performed a look-up of these previously associated hypertension or blood pressure variants in the current Japanese GWAS dataset.

\section{Gene and pathway-based analysis using VEGAS2 software}

We performed gene-based association testing using VEGAS2 (version 2) software56. VEGAS2 is an extension of the VErsatile Gene-based Association Study approach which uses 1000 genomes as reference data to estimate linkage disequilibrium between variants within a gene. Based on SNP association $P$ values of GWAS data, the software calculated empirical genebased $P$ values by a simulation procedure. We performed analysis using 1000 Genomes phase 3 East Asian populations (1000G EAS). Gene boundaries were set to $\pm 50 \mathrm{~kb}$ of each gene. Up to $10^{6}$ simulations were performed per gene. A total of 24,098 genes were tested, and genes with $P<2.07 \times 10^{-6}$ (Bonferroni correction for multiple testing, i.e., 0.05/24,098) were considered to be significantly associated with resistant hypertension. Subsequently, we performed pathway analysis using the VEGAS2Pathway approach57. VEGAS2Pathway performs pathway-based association testing and calculates empirical $P$-values of association for each pathway, while accounting for LD between variants within a gene and between neighboring genes, gene size, and pathway size by using a resampling of gene-based test statistics. The Biosystems gene-pathway annotation file was obtained from the VEGAS2 official site (https://vegas2.qimrberghofer.edu.au/biosystems20160324.vegas2pathSYM). The significance threshold of the empirical $P$-value in the pathway analysis was set at $1 \times 10^{-5}$ while taking into account the multiple testing of correlated pathways (0.05/5000 independent tests) 57.

\section{Data availability}

Individual genotyping data and clinical information that support the findings of this study are publicly available at the National Bioscience Database Center with accession code hum0014 (http://humandbs.biosciencedbc.jp/).

\section{Results}

\section{Genome-wide association studyfor resistant hypertension}

To clarify the genetic architecture of resistant hypertension, we conducted a GWAS in a Japanese population consisting of 2,705 resistant hypertension cases and 21,296 mild hypertension controls. We evaluated the possibility of population substructure for our sample population by comparison to HapMap samples using principal component analysis (PCA). Although all cases and controls were clustered in the Asian population, a very small portion of the samples was clustered in the Chinese population (Supplementary Fig. S1a and S1b). We then selected only samples from the major Japanese cluster for further analysis. After whole-genome imputation using the 1000 Genomes Projects as a reference, we examined the association of 6,012,291 SNPs with minor allele frequency (MAF) of more than $5 \%$ and an estimated imputation accuracy of greater than 0.8. The quantile-quantile (Q-Q) plot shows the distribution of observed versus expected $P$ values, while the corresponding genomic inflation factor $\left(\lambda_{G C}\right)$ of 1.057 suggests a low possibility of false-positive associations resulting from population stratification or cryptic relatedness (Supplementary Fig. S2). The Manhattan plot, plotting $-\log _{10}(P$ value $)$ from the GWAS and imputation analysis against the chromosome position, is shown in Fig. 1. Our GWAS identified one genetic locus achieving genome-wide significance $\left(P<5 \times 10^{-8}\right)$ and 17 loci showing suggestive association $\left(P<1 \times 10^{-5}\right)$ with resistant hypertension in the Japanese population (Table 1). We examined each locus for whether it included any variants that were previously reported to be significantly associated with blood pressure phenotypes, hypertension, or resistant hypertension in the $1 \mathrm{Mb}$ flanking region of each lead variant. We detected one novel locus with significant association and three novel loci with suggestive association. The lead variant of the novel significant locus was rs 1442386 (odds ratio $(95 \% \mathrm{Cl})=0.85(0.81-0.90)$, $P=3.75 \times 10^{-8}$ ), which is located in the intron region of DLG associated protein 1 (DLGAP1) on chromosome 18p11.3 (Fig. 2). The three novel suggestive loci were PQLC3 (2p25), LOC105369874 (12q14), and MED4 (13q14) locus. The other 14 suggestive 
loci included variants previously reported to be associated with hypertension or blood pressure phenotypes. For example, variants of the CYP11B2 locus have been frequently validated to be associated with hypertension in multiple populations 29,30 .

\section{Functional annotation and expression quantitative trait loci (eQTL) analysis}

We used HaploReg to perform functional analysis of a total of 18 lead variants showing association with resistant hypertension in the GWAS results. All of these variants were located in non-coding regions (nine intronic and nine intergenic) (Supplementary Table S2). Seven variants were located in gene expression regulatory motifs, such as enhancers, promoters, open chromatins and protein-binding sites in various tissue types. We found that several variants have been identified as eQTLs of their nearest genes in various tissue types (Supplementary Table S3). Among them, two variants including rs2075571 of 1q22 and rs9271382 of 6 p21 had associations $(P<0.05)$ with the expression levels of some genes, such as rs2075571 at the THBS3 locus showing an association with THBS3, GBA, RP11-263K19.6, GBAP1 and MUC1 expression, and rs9271382 at the HLADQA1 locus showing an association with HLA-DQA1, HLA-DQA2, HLA-DQB1, HLA-DQB1-AS1, HLA-DQB2, HLA-DRB1, HLA-DRB5, and $H L A-D R B 6$ expression in various tissue types. Functional analysis of the rs1442386 variant which reached genome-wide significance showed one altered regulatory motif (GCM; glia cells missing) and a significant association with DLGAP1 expression in whole blood at $P=0.0018$.

\section{Gene-based association analysis}

We used VEGAS2 to obtain gene-based $P$ values for phenotypic association from SNP-based $P$ values, using the 1000 Genomes Projects EAS phase 3 reference set. The genes of which the gene-based $P$ values exceeded a Bonferroni-corrected threshold of $P$ $<2.07 \times 10^{-6}$ are given in Supplementary Table S4. Gene-based tests identified 21 genes associated with resistant hypertension, including GBX1, AGAP3, ASB10, ABCF2 and TMUB1 on chromosome 7q36, ESRP1 and LOC100288748 on 8q22, CYP11B2, CYP11B, GML, LY6D, LYNX1_1, LYNX1_2 and LOC100133669 on 8q24, DLGAP1, DLGAP1-AS3, DLGAP1-AS4 and MIR6718 on 18p11, ERG on 21q22, and GAB4 and CECR7 on 22q11. These genes were located not only at one locus with significant association, but also at five loci with suggestive association, in the current GWAS. For four lead variants, rs 253447 of $5 q 31$, rs77163128 of 7p12, rs200741614 of 12q14, and rs11619475 of 13q14, no genes were identified by VEGAS2, because these variants were intergenic and were located over $50 \mathrm{~kb}$ outside the neighboring genes, resulting in them being outside the subject for gene-based association analysis.

\section{Pathway-based association analysis}

To further investigate the biological processes involved in resistant hypertension, we performed pathway-based association analysis using the VEGAS2Pathway approach. Figure 3 shows 35 Gene Ontology (GO) terms of biological process (BP) and cellular component (CC) that reached a genome-wide, pathway-based significant $P$ value of less than $1 \times 10^{-5}$ (Supplementary Table S5). Among them, we observed three prominent sets of GO terms that were highly associated with resistant hypertension. The most numerous set consisted of synapse (G0:0045202) and excitatory synapse (G0:0060076), especially involving postsynaptic compartments (CC term) for chemical synaptic transmission (G0:0007268) (BP term). Subsequently, a set of plasma membrane region (G0:0098590), postsynaptic membrane (G0:0045211), and membrane region (G0:0098589) for regulation of transmembrane transport (G0:0034762), and a set of neuron part (G0:0097458) and neuron projection (G0:0043005) for neuron development (G0:0048666) and neurological system process (G0:0050877) were also highly associated. These results suggest important pathways of the nervous system that may be involved in resistant hypertension.

\section{Evaluation of previously reported variants}

To verify previously reported loci showing an association with resistant hypertension, we performed analysis in the current Japanese GWAS dataset (Supplementary Table S6). These 26 SNPs have been previously evaluated in a multi-ethnic GWAS dataset including Caucasian, Hispanic, and African American subjects26-28,31. These SNPs, however, did not show a significant association with resistant hypertension in the Japanese population. We further examined whether variants previously associated with blood pressure phenotypes or hypertension showed an association with resistant hypertension in the current GWAS dataset. A total of 2074 and 172 SNPs were selected as variants previously associated with blood pressure and 
hypertension from the NHGRI-EBI GWAS catalog, respectively (listed in Supplementary Tables S7 and S8). Among these blood pressure-associated variants, eight at three loci showed suggestive associations with resistant hypertension $\left(P<1 \times 10^{-5}\right)$ (Supplementary Table S7). The most significant association was rs62525059 of 8q24 at the CYP11B2 locus $\left(P=2.58 \times 10^{-7}\right)$. The next suggestive associated variants were rs4072037 of 1q22 near MUC1/GBAP1 $\left(P=5.30 \times 10^{-6}\right)$ and rs3774427 of 3p21 near $C A C N A 1 D\left(P=6.51 \times 10^{-6}\right)$. Also, two loci, including rs62525059 (CYP11B2) and rs3774427 (CACNA1D), showed a suggestive association with resistant hypertension in variants previously associated with hypertension (Supplementary Table S8), the same as those previously associated with blood pressure. These results suggest the possibility that CYP11B2 (the aldosterone synthase gene) and CACNA1D (a member of the voltage-gated calcium channel gene family) may be involved in the development not only of hypertension, but also of resistant hypertension. However, the current GWAS data that were used to assess the association with resistant hypertension did not successfully replicate a large number of previous GWAS findings. Most of the previously reported variants associated with blood pressure were established from studies of quantitative traits of blood pressure phenotypes. In addition, previous GWASs of hypertension frequently adopted non-hypertensive subjects or general populations as the control. On the contrary, the present study evaluated a binary outcome using mild hypertensive controls, which may have led to a reduction in statistical power. These differences in our GWAS data may have resulted in the discrepancy in genetic correlations from previous findings in quantitative outcomes or studies using non-hypertensive controls.

\section{Discussion}

To investigate novel susceptibility loci for resistant hypertension, we performed a GWAS in the Japanese population consisting of 2,705 resistant hypertension cases and 21,296 controls. We identified a novel locus at chromosome 18p11.31 (DLGAP1) associated with resistant hypertension that reached genome-wide significance. Also, we identified 17 loci with suggestive association, 14 of which were previously reported to be associated with hypertension or blood pressure (e.g. the CYP11B2 locus).

The lead SNP (rs1442386) of the most significant association locus achieving genome-wide significance in this study was in the intron region of the DLGAP1 gene, which is exclusively expressed in brain and encodes disks large-associated protein 132. This variant is putatively GCM motif-altering, and is significantly associated with DLGAP1 expression in whole blood cells (Supplementary Table S2). The DLGAP1 protein, which localizes at postsynaptic density and interacts with postsynaptic density 95 (PSD95) protein, is involved in signaling at neuronal postsynaptic densities and maintaining normal brain function and development32. Although the findings of pathway analyses remain putative, our analyses showed that chemical synaptic transmission and regulation of transmembrane transport, including synaptic and trans-synaptic signaling pathways in the synapse and postsynaptic compartments, were significantly associated with resistant hypertension. Additionally, we observed significant associations of neuron development and neurological system process pathways with resistant hypertension. Our GWAS findings, combined with pathway analyses, provide an insight that the DLGAP1 protein at the postsynaptic membrane in the central nervous system may contribute to driving resistant hypertension, and highlight the importance of the nervous system in the etiology of resistant hypertension. Genetic variations of DLGAP1 are known to be associated with several psychiatric disorders, such as obsessive-compulsive disorder, schizophrenia, and major depressive disorder33-35. A worldwide epidemiological study has shown that resistant hypertension is associated with mental stress and anxiety 36 . These findings suggest that psychological stress may play a possible role in the pathophysiology of resistant hypertension. Further studies are needed to examine the associations between resistant hypertension and anxiety disorders and the possible roles of each in the development of the other.

The lead SNP (rs62525059) of the next suggestive association locus was located 9kb downstream of CYP11B2 (the aldosterone synthase gene), which is the rate limiting step of aldosterone synthesis in humans37. This variant was reported to be associated with blood pressure and hypertension in East Asian populations, including Japanese38,39. In addition, our replication study revealed that some variants, which were previously associated with both hypertension and blood pressure at the CYP11B2 locus, showed a suggestive association with resistant hypertension in our GWAS. Furthermore, gene-based association analysis in this study revealed that the CYP11B2 gene was significantly associated with resistant hypertension, supporting the GWAS results. Previous genetic studies of resistant hypertension revealed the association of variants related to 
the aldosterone and aldosterone pathways, including the beta and gamma subunits of the epithelial sodium channel ( $E N a C) 22,40$, angiotensinogen ( $A T G) 41$, and CYP4A1142. A recent study in Brazil showed that plasma aldosterone level was significantly associated with the $-344 \mathrm{C} / \mathrm{T}$ CYP11B2 polymorphism in 62 patients with resistant hypertension 25 . Regarding clinical features of resistant hypertension, excessive aldosterone is implicated in the pathophysiology of resistant hypertension11. Supporting this, elevation of circulating aldosterone level has been identified in the majority of patients with resistant hypertension11-13. Although previous GWASs for resistant hypertension in multi-ethnic populations did not detect any variant with a significant or suggestive association near the CYP11B2 region26-28,31, our findings in the Japanese population, in combination with previous genetic and clinical findings, suggest that the aldosterone synthase gene (CYP11B2) may be a potential causal gene for resistant hypertension, and support the important role of aldosterone and its pathways in the pathophysiology of resistant hypertension, the same as for hypertension.

Our GWAS data did not successfully replicate PTPRD findings in previous studies on resistant hypertension27,28. This discrepancy may derive in part from differences in race, selection of cases and controls, sample size of each study, and prescription rate for each class of antihypertensive drugs. In previous studies, discovery GWAS was performed using 1529 samples of genotype data of the INternational VErapamil-SR Trandolapril Study (INVEST)-GENEtic Substudy (INVEST-GENES), which collected DNA samples from INVEST study participants43. INVEST was a clinical trial evaluating adverse cardiovascular outcomes in multiple ethnic hypertensive patients with documented coronary artery disease who were randomly assigned to an atenolol-based $\beta$-blocker strategy or verapamil-SR-based calcium channel blocker strategy. The prescription rate for $\beta$-blockers in previous GWASs was approximately 50 percent in the non-resistant hypertension group from INVEST28. On the other hand, the prescription rate for $\beta$-blockers in this study was approximately 5 percent in the control group (Supplementary Table S9). It seems reasonable to speculate that the rate of patients with resistance to $\beta$-blockers in previous GWASs was higher than that in our GWAS. As the PTPRD gene is associated with blood pressure response to atenolol27, this difference in the prescription rate of $\beta$-blockers may be the reason that previous GWASs discovered an association with resistant hypertension in the PTPRD region, and that we could not find a susceptibility locus near the PTPRD gene.

Our study has some potential limitations. First, there is a possibility that diagnostic misclassification, which may impact on estimation of genetic correlations of GWAS44, may have occurred in our study. Because data on the diagnosis of hypertension were not available in this study, we defined patients with resistant hypertension based on antihypertensive drug prescription data. Also, data on blood pressure were limited to the first entry, resulting in incomplete evaluation of blood pressure control after the initiation of antihypertensive medications. Therefore, we adopted a more stringent diagnosis, defined as patients who had received four or more classes of drugs whatever their blood pressure control. However, this definition has a concern of potential systematic exclusion of uncontrolled patients who had received three classes of antihypertensive drugs. Second, regarding the control group, we identified patients with mild hypertension, defined as patients who had received one antihypertensive drug for at least one year. This definition may cause misclassification bias in that control samples may have included patients with moderate or severe hypertension, even if a small number of patients, leading to underestimation of genetic correlations. Also, we might have missed some positive results. These concerns call for further studies, such as replication studies using larger samples with diagnosis by physicians, to confirm the validity of our findings. Third, the ethnicity of all our study subjects was Japanese, and cases were ethnically matched with the controls, limiting the ability to generalize the results. Further studies are needed to confirm the association of the locus and resistant hypertension in other cohorts with different races, because the relevance of our findings to other ethnic groups remains to be demonstrated.

We identified a novel locus at the DLGAP1 gene with susceptibility to resistant hypertension with genome-wide significant levels, and 17 loci, including CYP11B2, as having suggestive association with resistant hypertension in the Japanese population. Pathway analysis revealed that chemical synaptic transmission, regulation of transmembrane transport, neuron development and neurological system processes are significantly associated with resistant hypertension. The DLGAP1 protein is known to be involved in signaling at neuronal postsynaptic densities and in maintaining normal brain function. Our novel findings suggest a possible role of $D L G A P 1$, which may contribute to susceptibility to resistant hypertension, possibly via the central nervous system, leading to a new target for drug discovery in the future. In addition, our data suggest that the aldosterone synthase gene 
(CYP11B2), which is known to be associated with hypertension, may be involved in the development not only of hypertension, but also of resistant hypertension, and underline the importance of this locus in resistant hypertension.

\section{Declarations}

\section{Acknowledgements}

We thank BioBank Japan for contributing to this study. BioBank Japan provided genotyping data and clinical information.

\section{Contributions}

Y.T. conceived and designed the study. K.Y. contributed to genomic analyses. Y.T. performed and interpreted bioinformatics analyses. K.M., M.K., and Y.K. contributed to the management of BBJ data. S.A. supervised this study. Y.T. wrote the manuscript with critical input from K.M. and Y.K.

\section{Ethics declarations}

\section{Competing interests}

The authors declare no competing interests.

\section{References}

1. Chobanian, A.V. et al. The Seventh Report of the Joint National Committee on Prevention, Detection, Evaluation, and Treatment of High Blood Pressure: the JNC 7 report. JAMA289, 2560-72 (2003).

2. Calhoun, D.A. et al. Resistant hypertension: diagnosis, evaluation, and treatment: a scientific statement from the American Heart Association Professional Education Committee of the Council for High Blood Pressure Research. Circulation117, e510-26 (2008).

3. Major outcomes in high-risk hypertensive patients randomized to angiotensin-converting enzyme inhibitor or calcium channel blocker vs diuretic: The Antihypertensive and Lipid-Lowering Treatment to Prevent Heart Attack Trial (ALLHAT). JAMA288, 2981-97 (2002).

4. Gupta, A.K. et al. Baseline predictors of resistant hypertension in the Anglo-Scandinavian Cardiac Outcome Trial (ASCOT): a risk score to identify those at high-risk. J Hypertens29, 2004-13 (2011).

5. Egan, B.M., Zhao, Y., Axon, R.N., Brzezinski, W.A. \& Ferdinand, K.C. Uncontrolled and apparent treatment resistant hypertension in the United States, 1988 to 2008. Circulation124, 1046-58 (2011).

6. Sarafidis, P.A., Georgianos, P. \& Bakris, G.L. Resistant hypertension-its identification and epidemiology. Nat Rev Nephro/9, 51-8 (2013).

7. Vongpatanasin, W. Resistant hypertension: a review of diagnosis and management. JAMA311, 2216-24 (2014).

8. Daugherty, S.L. et al. The association between medication adherence and treatment intensification with blood pressure control in resistant hypertension. Hypertension60, 303-9 (2012).

9. Shimbo, D. et al. The contributions of unhealthy lifestyle factors to apparent resistant hypertension: findings from the Reasons for Geographic And Racial Differences in Stroke (REGARDS) study. J Hypertens31, 370-6 (2013).

10. De Nicola, L. et al. Prevalence and prognostic role of resistant hypertension in chronic kidney disease patients. J Am Coll Cardio/61, 2461-2467 (2013). 
11. Gaddam, K.K. et al. Characterization of resistant hypertension: association between resistant hypertension, aldosterone, and persistent intravascular volume expansion. Arch Intern Med168, 1159-64 (2008).

12. de Haro Moraes, C. et al. High-circulating leptin levels are associated with increased blood pressure in uncontrolled resistant hypertension. J Hum Hypertens27, 225-30 (2013).

13. de Faria, A.P. et al. Hypoadiponectinemia and aldosterone excess are associated with lack of blood pressure control in subjects with resistant hypertension. Hypertens Res36, 1067-72 (2013).

14. Duprez, D.A. Aldosterone and the vasculature: mechanisms mediating resistant hypertension. J Clin Hypertens (Greenwich)9, 13-8 (2007).

15. El Rouby, N. \& Cooper-DeHoff, R.M. Genetics of resistant hypertension: a novel pharmacogenomics phenotype. Curr Hypertens Rep17, 583 (2015).

16. Levy, D. et al. Genome-wide association study of blood pressure and hypertension. Nat Genet41, 677-87 (2009).

17. Newton-Cheh, C. et al. Genome-wide association study identifies eight loci associated with blood pressure. Nat Genet41, 666-76 (2009).

18. Franceschini, N., Reiner, A.P. \& Heiss, G. Recent findings in the genetics of blood pressure and hypertension traits. Am J Hypertens 24, 392-400 (2011).

19. Binder, A. A review of the genetics of essential hypertension. Curr Opin Cardio/22, 176-84 (2007).

20. Cruz-Gonzalez, I. et al. An association between resistant hypertension and the null GSTM1 genotype. J Hum Hypertens23, 556-8 (2009).

21. Cruz-Gonzalez, I. et al. Association between -T786C NOS3 polymorphism and resistant hypertension: a prospective cohort study. BMC Cardiovasc Disord9, 35 (2009).

22. Hannila-Handelberg, T. et al. Common variants of the beta and gamma subunits of the epithelial sodium channel and their relation to plasma renin and aldosterone levels in essential hypertension. BMC Med Genet6, 4 (2005).

23. Ho, H. et al. Association between the CYP3A5 genotype and blood pressure. Hypertension45, 294-8 (2005).

24. Jachymova, M. et al. Association of the Glu298Asp polymorphism in the endothelial nitric oxide synthase gene with essential hypertension resistant to conventional therapy. Biochem Biophys Res Commun284, 426-30 (2001).

25. Fontana, V. et al. Modulation of aldosterone levels by -344 C/T CYP11B2 polymorphism and spironolactone use in resistant hypertension. J Am Soc Hypertens8, 146-51 (2014).

26. Dumitrescu, L. et al. Genome-wide study of resistant hypertension identified from electronic health records. PLoS One12, e0171745 (2017).

27. Gong, Y. et al. PTPRD gene associated with blood pressure response to atenolol and resistant hypertension. J Hypertens33, 2278-85 (2015).

28. El Rouby, N. et al. Genome-wide association analysis of common genetic variants of resistant hypertension. Pharmacogenomics J19, 295-304 (2018).

29. Alvarez-Madrazo, S. et al. Common polymorphisms in the CYP11B1 and CYP11B2 genes: evidence for a digenic influence on hypertension. Hypertension61, 232-9 (2013). 
30. Zhang, H. et al. A novel haplotype of low-frequency variants in the aldosterone synthase gene among northern Han Chinese with essential hypertension. Medicine (Baltimore)96, e8150 (2017).

31. Irvin, M.R. et al. Genome-wide association study of apparent treatment-resistant hypertension in the CHARGE Consortium: The CHARGE Pharmacogenetics Working Group. Am J Hypertens32, 1146-1153 (2019).

32. Kawashima, N., Takamiya, K., Sun, J., Kitabatake, A. \& Sobue, K. Differential expression of isoforms of PSD-95 binding protein (GKAP/SAPAP1) during rat brain development. FEBS Lett418, 301-4 (1997).

33. Stewart, S.E. et al. Genome-wide association study of obsessive-compulsive disorder. Mol Psychiatry18, 788-98 (2013).

34. Li, J.M. et al. Genetic analysis of the DLGAP1 gene as a candidate gene for schizophrenia. Psychiatry Res205, 13-7 (2013).

35. Mathias, S.R. et al. Recurrent major depression and right hippocampal volume: A bivariate linkage and association study. Hum Brain Mapp37, 191-202 (2016).

36. Schmieder, R.E., Grassi, G. \& Kjeldsen, S.E. Patients with treatment-resistant hypertension report increased stress and anxiety: a worldwide study. J Hypertens31, 610-5; discussion 615 (2013).

37. Strushkevich, N. et al. Structural insights into aldosterone synthase substrate specificity and targeted inhibition. Mol Endocrino/27, 315-24 (2013).

38. Takeuchi, F. et al. Interethnic analyses of blood pressure loci in populations of East Asian and European descent. Nat Commun9, 5052 (2018).

39. Kanai, M. et al. Genetic analysis of quantitative traits in the Japanese population links cell types to complex human diseases. Nat Genet50, 390-400 (2018).

40. Jones, E.S., Owen, E.P. \& Rayner, B.L. The association of the R563Q genotype of the ENaC with phenotypic variation in Southern Africa. Am J Hypertens25, 1286-91 (2012).

41. Yugar-Toledo, J.C. et al. Gene variation in resistant hypertension: multilocus analysis of the angiotensin 1-converting enzyme, angiotensinogen, and endothelial nitric oxide synthase genes. DNA Cell Bio/30, 555-64 (2011).

42. Laffer, C.L. et al. Genetic variation in CYP4A11 and blood pressure response to mineralocorticoid receptor antagonism or ENaC inhibition: an exploratory pilot study in African Americans. J Am Soc Hypertens8, 475-80 (2014).

43. Pepine, C.J. et al. A calcium antagonist vs a non-calcium antagonist hypertension treatment strategy for patients with coronary artery disease. The International Verapamil-Trandolapril Study (INVEST): a randomized controlled trial. JAMA290, 2805-16 (2003).

44. Wray, N.R., Lee, S.H. \& Kendler, K.S. Impact of diagnostic misclassification on estimation of genetic correlations using genome-wide genotypes. Eur J Hum Genet20, 668-74 (2012).

45. Nagai, A. et al. Overview of the BioBank Japan Project: Study design and profile. J Epidemio/27, S2-S8 (2017).

46. Hirata, M. et al. Cross-sectional analysis of BioBank Japan clinical data: A large cohort of 200,000 patients with 47 common diseases. J Epidemiol27, S9-S21 (2017).

47. Purcell, S. et al. PLINK: a tool set for whole-genome association and population-based linkage analyses. Am J Hum Genet81, 559-75 (2007).

48. Price, A.L. et al. Principal components analysis corrects for stratification in genome-wide association studies. Nat Genet38, 904-9 (2006). 
49. Devlin, B. \& Roeder, K. Genomic control for association studies. Biometrics55, 997-1004 (1999).

50. Delaneau O, Marchini J, Zagury JF. A linear complexity phasing method for thousands of genomes. Nat Methods. 2012 Dec 4;9(2):179-81.

51. Howie, B.N., Donnelly, P. \& Marchini, J. A flexible and accurate genotype imputation method for the next generation of genome-wide association studies. PLoS Genet5, e1000529 (2009).

52. Auton, A. et al. A global reference for human genetic variation. Nature526, 68-74 (2015)

53. Marchini, J., Howie, B., Myers, S., McVean, G. \& Donnelly, P. A new multipoint method for genome-wide association studies by imputation of genotypes. Nat Genet39, 906-13 (2007).

54. Pruim, R.J. et al. LocusZoom: regional visualization of genome-wide association scan results. Bioinformatics26, 2336-7 (2010).

55. Ward, L.D. \& Kellis, M. HaploReg: a resource for exploring chromatin states, conservation, and regulatory motif alterations within sets of genetically linked variants. Nucleic Acids Res40, D930-4 (2012).

56. Mishra, A. \& Macgregor, S. VEGAS2: Software for more flexible gene-based testing. Twin Res Hum Genet18, 86-91 (2015).

57. Mishra, A. \& MacGregor, S. A novel approach for pathway analysis of GWAS data highlights role of BMP signaling and muscle cell differentiation in colorectal cancer susceptibility. Twin Res Hum Genet20, 1-9 (2017).

58. BioBank Japan, https://biobankjp.org/english/index.html; PLINK, http://zzz.bwh.harvard.edu/plink/; smartpca, http://genepath.med.harvard.edu/ reich/Software.htm; SHAPEIT, https://mathgen.stats.ox.ac.uk/genetics_software/shapeit/shapeit.html; IMPUTE2, http://mathgen.stats.ox.ac.uk/impute/impute_v2.html; 1000 Genomes Projects, http://www.1000genomes.org/; R, https://www.r-project.org/; HaploReg, http://www.broadinstitute.org/mammals/haploreg/haploreg.php; LocusZoom, http://locuszoom.sph.umich.edu/locuszoom/; VEGAS2, https://vegas2.qimrberghofer.edu.au/; GWAS Catalogue, https://www.ebi.ac.uk/gwas/; NCBI SNP database, https://www.ncbi.nlm.nih.gov/snp/

\section{Tables}

Table 1. Summary of association results of genome-wide association study in Japanese population. 


\begin{tabular}{|c|c|c|c|c|c|c|c|c|c|c|c|}
\hline SNP & Chr. & Position & Nearest gene & RA & NRA & MAF & & OR & L95 & U95 & $P$ \\
\hline & & (bp) & & & & Cases & Controls & & & & \\
\hline rs2075571 & 1 & 155204315 & THBS3 & C & $\mathrm{T}$ & 0.211 & 0.184 & 0.84 & 0.79 & 0.90 & $\begin{array}{l}1.24 \mathrm{E}- \\
06\end{array}$ \\
\hline rs3732103* & 2 & 11172098 & PQLC3 & C & $\mathrm{T}$ & 0.436 & 0.470 & 0.87 & 0.82 & 0.92 & $\begin{array}{l}1.94 \mathrm{E}- \\
06\end{array}$ \\
\hline rs7625237 & 3 & 53247249 & $T K T$ & $\mathrm{~T}$ & C & 0.464 & 0.499 & 1.15 & 1.09 & 1.22 & $\begin{array}{l}1.15 \mathrm{E}- \\
06\end{array}$ \\
\hline rs253447 & 5 & 142412468 & SPRY4 & C & $\mathrm{T}$ & 0.493 & 0.460 & 0.88 & 0.83 & 0.93 & $\begin{array}{l}6.08 \mathrm{E}- \\
06\end{array}$ \\
\hline rs9271382 & 6 & 32619598 & $H L A-D Q A 1$ & G & $A$ & 0.460 & 0.495 & 0.87 & 0.82 & 0.92 & $\begin{array}{l}9.73 \mathrm{E}- \\
07\end{array}$ \\
\hline rs77163128 & 7 & 46063047 & IGFBP3 & $\mathrm{T}$ & G & 0.286 & 0.257 & 1.16 & 1.09 & 1.23 & $\begin{array}{l}1.93 \mathrm{E}- \\
06\end{array}$ \\
\hline rs79549409 & 7 & 151157081 & $G B X 1$ & $A$ & G & 0.113 & 0.136 & 0.81 & 0.74 & 0.88 & $\begin{array}{l}1.22 \mathrm{E}- \\
06\end{array}$ \\
\hline rs4247284 & 8 & 94647408 & ESRP1 & CA & G & 0.276 & 0.245 & 0.85 & 0.80 & 0.91 & $\begin{array}{l}8.85 \mathrm{E}- \\
07\end{array}$ \\
\hline rs62525059 & 8 & 142901545 & CYP11B2 & G & $A$ & 0.335 & 0.371 & 0.85 & 0.80 & 0.91 & $\begin{array}{l}2.58 \mathrm{E}- \\
07\end{array}$ \\
\hline rs10833346 & 11 & 20522091 & PRMT3 & $A$ & G & 0.152 & 0.178 & 0.83 & 0.77 & 0.90 & $\begin{array}{l}2.47 \mathrm{E}- \\
06\end{array}$ \\
\hline rs9787901 & 11 & 45674603 & CHST1 & G & $A$ & 0.386 & 0.418 & 0.87 & 0.83 & 0.93 & $\begin{array}{l}6.25 \mathrm{E}- \\
06\end{array}$ \\
\hline rs200741614* & 12 & 83369808 & LOC105369874 & G & $\mathrm{T}$ & 0.096 & 0.078 & 1.25 & 1.14 & 1.38 & $\begin{array}{l}1.89 \mathrm{E}- \\
06\end{array}$ \\
\hline rs11619475* & 13 & 48151713 & MED4 & $\mathrm{T}$ & C & 0.366 & 0.396 & 1.14 & 1.07 & 1.21 & $\begin{array}{l}5.13 \mathrm{E}- \\
06\end{array}$ \\
\hline rs73324844 & 17 & 61064318 & $B C A S 3$ & $A$ & C & 0.145 & 0.122 & 1.22 & 1.12 & 1.32 & $\begin{array}{l}1.64 \mathrm{E}- \\
06\end{array}$ \\
\hline rs1442386* & 18 & 3938439 & $D L G A P 1$ & A & G & 0.445 & 0.484 & 0.85 & 0.81 & 0.90 & $\begin{array}{l}3.75 \mathrm{E}- \\
08\end{array}$ \\
\hline rs117652372 & 18 & 55607167 & TCF4 & G & $A$ & 0.100 & 0.083 & 1.22 & 1.11 & 1.35 & $\begin{array}{l}3.14 \mathrm{E}- \\
06\end{array}$ \\
\hline rs2212606 & 21 & 38673016 & $E R G$ & $A$ & C & 0.298 & 0.333 & 0.85 & 0.80 & 0.91 & $\begin{array}{l}4.48 \mathrm{E}- \\
07\end{array}$ \\
\hline rs78813487 & 22 & 17007603 & GAB4 & $\mathrm{T}$ & C & 0.071 & 0.056 & 1.29 & 1.18 & 1.45 & $\begin{array}{l}6.18 \mathrm{E}- \\
07\end{array}$ \\
\hline
\end{tabular}

SNP, single-nucleotide polymorphism (rsID of lead SNP); Chr; chromosome, Position, physical position of human genome version of GRCh38; RA; risk allele, NRA; non-risk allele, OR; odds ratio, L95; lower 95\% confidence limit, U95; upper 95\% confidence limit, MAF; minor allele frequency.

Odds ratios $(\mathrm{OR})$ and confidence intervals $(\mathrm{Cl})$ were calculated using the non-risk allele as a reference. *: indicates a novel locus for resistant hypertension. The nearest gene is shown as the locus label, but should not be interpreted as the best candidate. A list of all the genes in the 500-kb flanking region of the lead SNP is presented in Table S1.

Table 2. Baseline characteristics of study population. 


\begin{tabular}{|lll|}
\hline & Cases & Controls \\
\hline Number of samples & 2,705 & 21,296 \\
\hline Women $(\%)$ & $35.8 \%$ & $42.1 \%$ \\
\hline Age (years) & $65.8 \pm 10.5$ & $66.4 \pm 10.9$ \\
\hline BMI $\left(\mathrm{kg} / \mathrm{m}^{2}\right)$ & $25.4 \pm 4.07$ & $23.6 \pm 3.5$ \\
\hline Blood pressure $(\mathrm{mmHg})$ & & \\
\hline Systolic & $139.9 \pm 18.5$ & $131.5 \pm 15.6$ \\
\hline Diastolic & $79.1 \pm 12.4$ & $76.5 \pm 10.5$ \\
\hline
\end{tabular}

Note: Data are mean \pm SD unless otherwise stated.

Abbreviations: BMI, body mass index; SD, standard deviation.

\section{Figures}

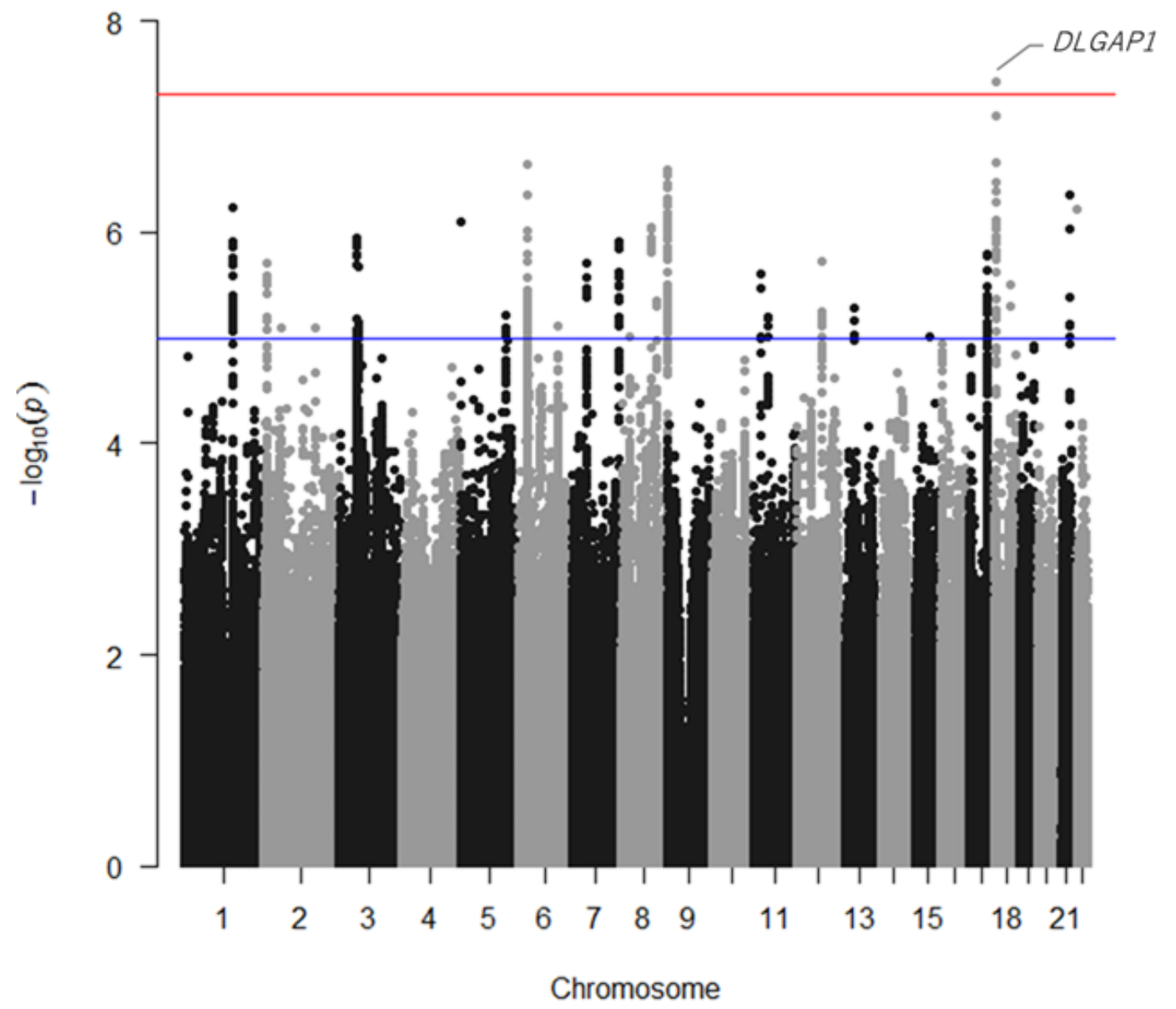

Figure 1

Manhattan plot for genome-wide association study (GWAS) of resistant hypertension in Japanese population. This plot is based on -log10(P value) from GWAS and imputation analysis against chromosome position. Blue line indicates suggestive association threshold, $\mathrm{P}<1 \times 10-5$; red line indicates genome-wide significance threshold, $\mathrm{P}<5 \times 10-8$. 


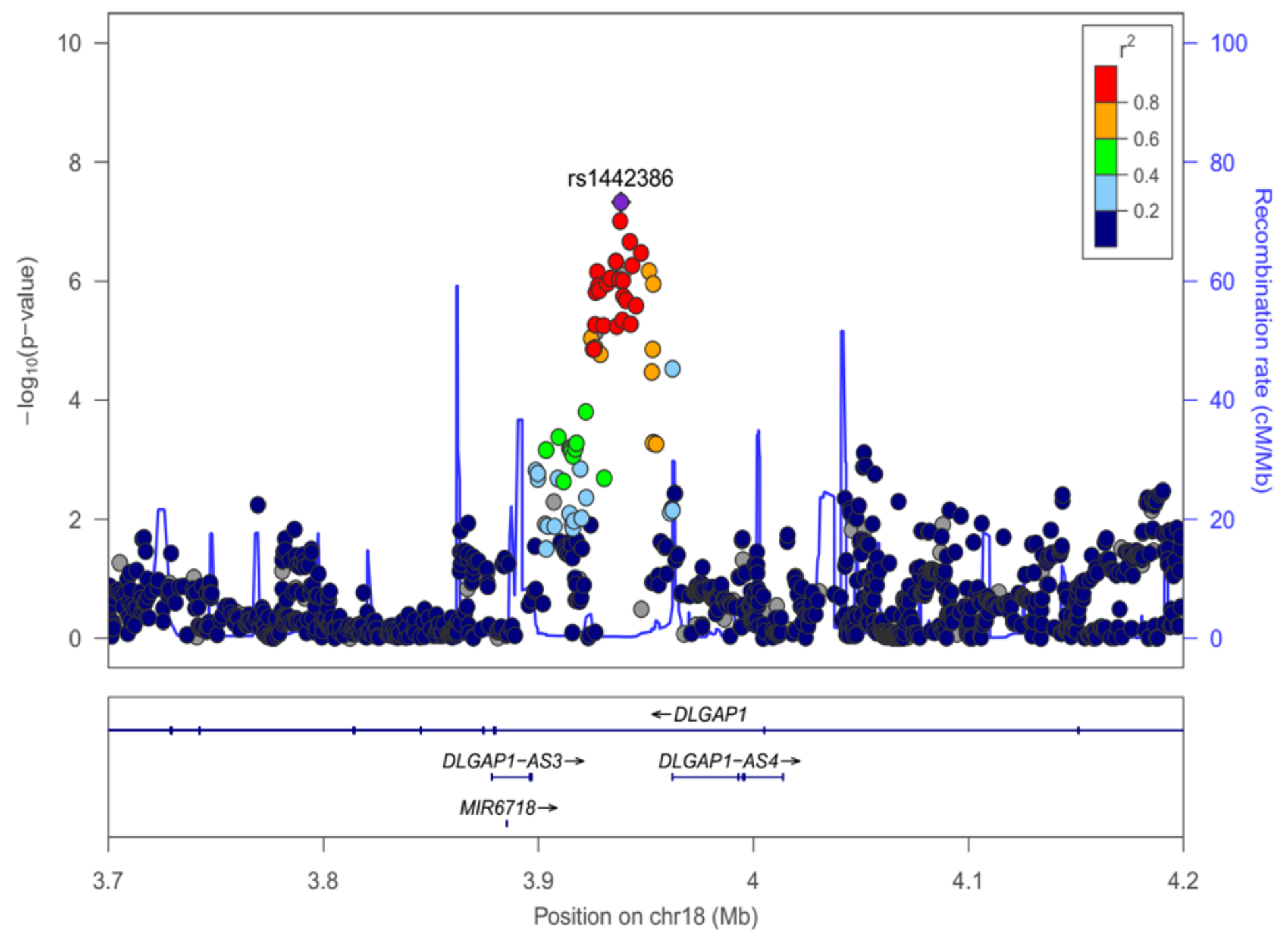

Figure 2

Regional association plots for locus significantly associated with resistant hypertension in Japanese population, on chromosome 18p11.3 (DLGAP1). The regional association plot was generated from the association data of a genome-wide association study (GWAS) with LocusZoom. SNPs are colored according to their LD with the marker SNP: red ( $r 2 \geq 0.8)$, orange $(0.6 \leq \mathrm{r} 2 \leq 0.8)$, green $(0.4 \leq \mathrm{r} 2 \leq 0.6)$, light blue $(0.2 \leq \mathrm{r} 2 \leq 0.4)$, dark blue $(r 2<0.2)$. Purplish blue lines represent local recombination rates. Diamonds (rs1442386) represent the most significantly associated SNP in each region in the GWAS. The SNP position is based on NCBI build 37. 


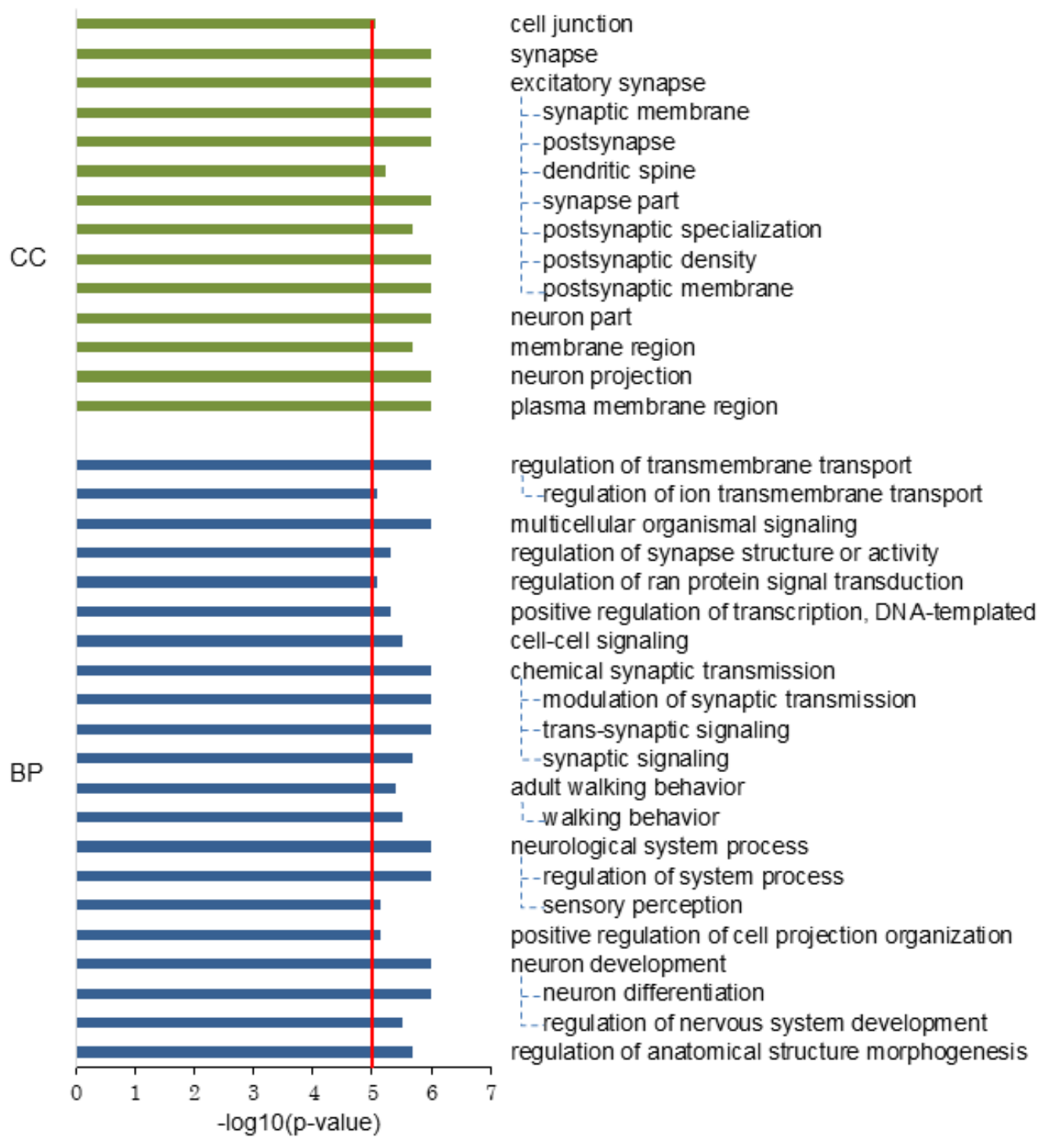

\section{Figure 3}

Results of significant pathway for resistant hypertension. Pathway analysis by VEGAS2Pathway revealed 35 significant pathways in cellular component (CC) (green) and biological process (BP) (blue). Red line indicates significance threshold of pathway-based $P$ value $(P<1 \times 10-5)$.

\section{Supplementary Files}

This is a list of supplementary files associated with this preprint. Click to download.

- SupplementaryFiguresv6SR.docx

- SupplementaryTablesv6SR.xlsx 